\title{
A New Method for Temperature Spatial Interpolation Based on Sparse Historical Stations ${ }^{\mathbb{O}}$
}

\author{
CHENGDONG XU AND Jinfeng WANG \\ State Key Laboratory of Resources and Environmental Information System, Institute of Geographic Sciences \\ and Natural Resources Research, Chinese Academy of Sciences, Beijing, China \\ QINGXIANG LI \\ School of Atmospheric Sciences, Sun Yat-sen University, Guangzhou, China
}

(Manuscript received 6 March 2017, in final form 10 November 2017)

\begin{abstract}
Long-term grid historical temperature datasets are the foundation of climate change research. Datasets developed by traditional interpolation methods usually contain data for a period of less than $50 \mathrm{yr}$, with a relatively low spatial resolution owing to the sparse distribution of stations in the historical period. In this study, the point interpolation based on Biased Sentinel Hospitals Areal Disease Estimation (P-BSHADE) method has been used to interpolate 1-km grids of monthly surface air temperatures in the historical period of 1900-50 in China. The method can be used to remedy the station bias resulting from sparse coverage, and it considers the characteristics of spatial autocorrelation and nonhomogeneity of the temperature distribution to obtain unbiased and minimum error variance estimates. The results have been compared with those from widely used methods such as kriging, inverse distance weighting (IDW), and a combined spline with kriging (TPS-KRG) method, both theoretically and empirically. The leave-one-out cross-validation method using a real dataset was implemented. The root-mean-square error (RMSE) [mean absolute error (MAE)] for P-BSHADE is $0.98^{\circ} \mathrm{C}\left(0.75^{\circ} \mathrm{C}\right)$, while those for TPS-KRG, kriging, and IDW are $1.46^{\circ}\left(1.07^{\circ}\right)$, $2.23^{\circ}\left(1.51^{\circ}\right)$, and $2.64^{\circ} \mathrm{C}\left(1.85^{\circ} \mathrm{C}\right)$, respectively. The results of validation using a simulated dataset also present the smallest error for P-BSHADE, demonstrating its empirical superiority. In addition to its empirical superiority, the method also can produce a map of the estimated error variance, representing the uncertainty of estimation.
\end{abstract}

\section{Introduction}

In the last century, global temperature has experienced a rapid increase, and there have been immediate demands for research on the characteristics of climate change and its influence on human society and the ecosystem. Longterm historical temperature datasets are the foundation for climate change research (Cao et al. 2016; Jones and Lister 2009; Xu et al. 2017).

Observation records from meteorological stations are the most widely used datasets for studies on climate change, compared to other datasets (e.g., tree-ring data).

Supplemental information related to this paper is available at the Journals Online website: https://doi.org/10.1175/JCLI-D-170150.s1.

Corresponding author: Jinfeng Wang, wangjf@lreis.ac.cn
They have advantages owing to their high reliability and accuracy. Observation stations in historical periods are sparse and unevenly distributed (Li et al. 2010, 2017; Cao et al. 2013); therefore, in studies on climate change, varieties of gridded historical climate data at regional and global scales have been created (DeGaetano and Belcher 2007; Fan and van den Dool 2008; Hijmans et al. 2005; Di Luzio et al. 2008; Mitchell and Jones 2005). The grid datasets have the advantage of being easily used in mapping and as input data in many models, and they are widely used by researchers in related fields at the global and regional scales (Daly 2006; Li et al. 2017).

There are two types of interpolation approaches for creating gridded datasets. One type includes grid-based methods, which aggregate the local station observations into average values on a regular grid; the other type uses station records to interpolate temperature at target locations (e.g., grid points) based on various models. 
Based on grid-based methods and instrumental records, several datasets of longstanding global land surface air temperatures have been developed and widely used. Three major players in this field are the UK Met Office Hadley Centre and the University of East Anglia Climatic Research Unit (HadCRUT), NOAA's National Center for Environmental Information (NCEI), and the NASA Goddard Institute for Space Studies (GISS) (Jones 2016).

The CRUTEM4 dataset has been updated by HadCRUT (Jones et al. 2012). The Global Historical Climatology Network Version 3 (GHCNv3) dataset has been updated by NCEI (formerly the National Climatic Data Center) (Lawrimore et al. 2011). These two gridded datasets are produced by the climate anomaly method (CAM) (Jones 1994). CAM is simple and straightforward and is widely used in the creation of gridded datasets. However, when a station does not have enough data for the reference period, the data of the station from the historical period cannot be used. The gridded datasets from GISS are produced using the reference station method (RSM) (Hansen and Lebedeff 1987). This method can employ all station data in the historical period under the assumption that the reference station can represent the true climate characteristics in the grid box where it is located.

These global gridded datasets agree well with each other for the recent decades when a large number of stations have been present (Hansen et al. 2010; Hartmann et al. 2013); however, before the early part of the last century, there were significant discrepancies between the datasets, although similar station data were employed in their estimation (Jones and Wigley 2010). One of the most important reasons for these discrepancies is the sparse distribution of the stations in the early years, which would introduce uncertainty in the process of gridded temperature interpolation (Brohan et al. 2006; Hansen et al. 2010; Jones and Wigley 2010; Jones et al. 2012).

Besides the abovementioned grid-based methods, various station-based methods are used to produce gridded datasets; the commonly used methods are regression-based, distance-weighted, spline, and geostatistical methods. They are often used in combination with different methods.

Regression-based methods are widely used in climate data interpolation in which the temperature at the target location is estimated using the linear relationship between temperature and explanatory variables (Daly 2006; Eischeid et al. 2000; Stahl et al. 2006). For example, Daly et al. (2003) applied the Parameter-Elevation Regressions on Independent Slopes Model (PRISM) to generate maps of mean monthly and annual minimum and maximum temperatures for the Caribbean island of
Puerto Rico coving an area of about $8740 \mathrm{~km}^{2}$ using 108 stations for the period 1963-95 (Daly et al. 2003). Guan et al. (2013) used a multiple-regression model to interpolate the spatial distribution of mean monthly daily maximum and minimum temperatures using 33 stations in an area of $9000 \mathrm{~km}^{2}$ in Adelaide and the Mount Lofty Ranges in South Australia.

Distance-weighted methods, such as inverse distance weighting (IDW) (Di Piazza et al. 2011), only use the climatic data from meteorological stations. The temperature at a target location is calculated as a weighted average of observations from neighboring stations, and the weight decreases as the distance between the target location and the neighboring observation stations increases. Using IDW, Piper developed the first global land surface $1^{\circ} \times 1^{\circ}$ gridded dataset of average daily temperatures for 1987 (Piper and Stewart 1996). Di Luzio et al. (2008) combined IDW and PRISM to interpolate the daily temperatures for the conterminous United States at spatial resolution of $2.5 \mathrm{~min}$ for the period 1960-2001. Nalder and Wein (1998) introduced a method named the gradient-plus-inverse distance squared (GIDS) method, which combines multiple linear regression and distance weighting techniques. This method has been tested by interpolating monthly temperatures in western Canada using 32 stations from 1961 to 1990 , showing that the method is superior to all kinds of kriging methods for the study area.

Spline methods can obtain an estimated surface having maximizing smoothness by using a nonlinear function. The main strength of spline methods is their relatively simple calculation, as fewer parameters are required. Hijmans et al. (2005) used a spline method to interpolate a monthly global land surface climate dataset at a 1-km spatial resolution from 1950 to 2000. New et al. (1999) used a spline method to construct a $0.5^{\circ} \times$ $0.5^{\circ}$ global land surface climatology dataset for the period 1961-90, by including the mean temperature and other variables. In the interpolation process, the covariables of latitude, longitude, and elevation were used.

Many methods belonging to the family of geostatistical methods (Goovaerts 1997; Isaaks and Srivastava 1989; Olea 1999) have been used in various applications for the interpolation of climate data (Boer et al. 2001; Hudson and Wackernagel 1994; Jeffrey et al. 2001). These methods have the assumption of second-order stationary; the mathematical expectation of temperature variables is assumed to be constant in any location and the relationship of the temperature between two locations is determined only by the spatial distance (Burrough and McDonnell 1998). Hofstra et al. (2008) interpolated the European area temperature from 1961 to 1990 and found that the kriging 
method performed the best. Haylock et al. (2008) interpolated daily temperatures with $0.25^{\circ}$ and $0.5^{\circ}$ resolutions by using the kriging and spline methods with the European Climate Assessment and Dataset from 1950 to 2006. Kilibarda et al. (2014) produced a gridded dataset of daily air temperature using a spatiotemporal regression-kriging method at a spatial resolution of $1 \mathrm{~km}$. The cross validation shows that although there is no significant reduction of the estimated error compared with splines, the kriging method has an advantage of allowing the quantification of uncertainty.

The datasets developed by the above four stationbased interpolation methods usually do not cover more than $50 \mathrm{yr}$ and have relatively coarse spatial resolutions (e.g., a few degrees in latitude and longitude) for most of the studies. This is attributed to the sparse distribution of stations in the early part of the historical period.

The sparse coverage of stations will introduce uncertainty in the temperature interpolation, as small samples are biased to a large area with stratified heterogeneity (Wang et al. 2014). In addition, spatial autocorrelation and nonhomogeneity are pervasive in the temperature field on Earth's surface. If this information were employed, the interpolation precision would be improved (Christakos 1992; Goovaerts 1997; Haining 2003). However, the traditional methods do not consider the bias of samples and do not take into account the characteristics of spatial autocorrelation and nonhomogeneity simultaneously.

To solve the problem, the point interpolation based on Biased Sentinel Hospitals Areal Disease Estimation (P-BSHADE) method has been developed, which was proposed by Wang et al. (2011) originally in the area of health geography. P-BSHADE can be used to remedy the station bias resulting from sparse coverage since it considers the characteristics of spatial autocorrelation and nonhomogeneity of the temperature distribution. It has been used to interpolate missing data in climate datasets and estimate regional mean temperatures, and the results show that it yields a more precise estimation than other commonly used methods in the historical period with sparse station coverage (Wang et al. 2014; C.-D. $\mathrm{Xu}$ et al. 2013). However, it cannot interpolate climate variables to anywhere except the locations with missing data.

The objective of the present study is to develop an interpolation method to produce a $1-\mathrm{km}$ grid dataset using sparse station coverage based on P-BSHADE in the historical period. This method can be used to interpolate with no limitation to where station data exists. The performance of the method will be compared with that of other widely used methods. This

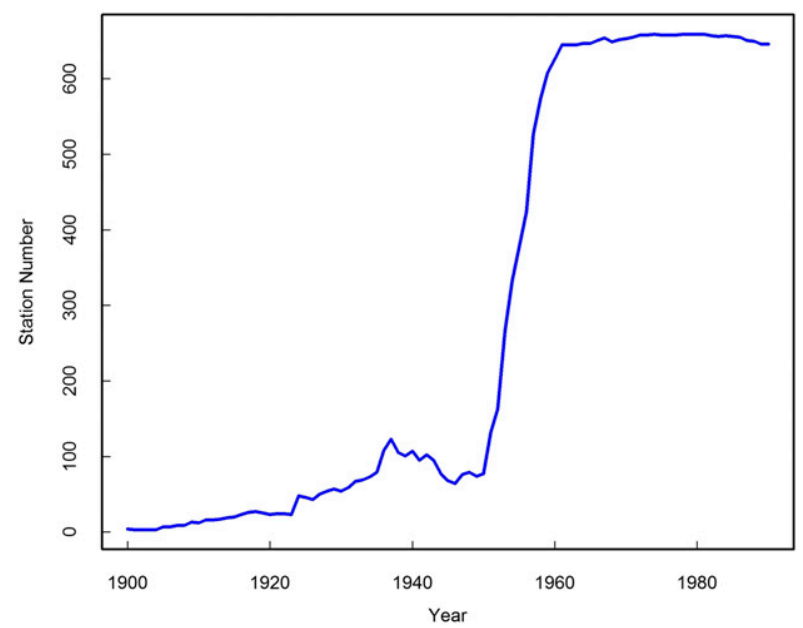

FIG. 1. Number of stations in China from 1900 to 1990.

method is expected to provide an efficient climate interpolation even for the early part of the historical period.

\section{Data and methods}

a. Station data

Several homogenized in situ datasets have been provided for analysis of the interpolation of the surface air temperature data during recent years ( $\mathrm{Li}$ et al. 2010, 2017; W. Xu et al. 2013; Cao et al. 2013, 2017). Here, a temperature station dataset of monthly averages from 1900 to 1990 was used for spatial interpolation. This dataset was constructed by the National Meteorological Information Centre of China, and the temperature records in the dataset have has been quality controlled and homogenized (Li et al. 2010, 2017; W. Xu et al. 2013). Prior to the 1950s, the spatial distribution of stations was very sparse, with the stations being mainly located in eastern China, in the political and economic center with a dense population. The number of stations increased sharply after the establishment of the People's Republic of China in 1949. In the 1960s, the density of stations reached the highest level, where it currently remains today (Figs. 1 and 2).

\section{b. DEM data}

The digital elevation model (DEM) data used in this study are taken from DEMSRE3, which is a 1-km grid solution and is obtained from the WorldGrids.org portal (http://worldgrids.org/doku.php/wiki:demsre3). The dataset is derived as a combination of the Shuttle Radar Topography Mission 30 (SRTM30) and ETOPO data. In this study, the DEMSRE3 data were used to extract the elevation values of stations and locations of each 

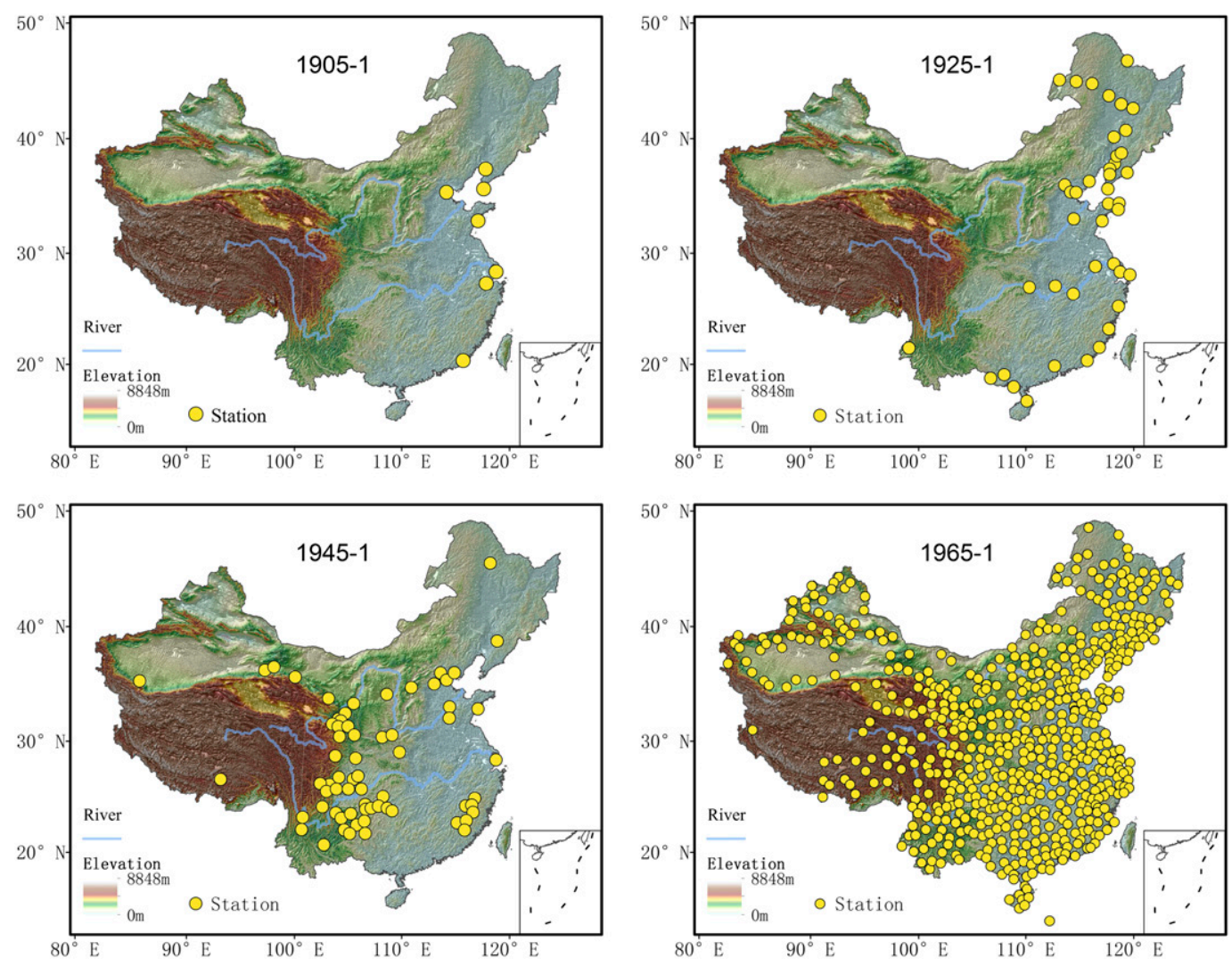

FIG. 2. Distributions of stations in January from 1905 to 1965 in China, a span of 60 years.

grid, and then used to produce the climatological field of the surface air temperature in the reference period as an explanatory variable, which will be finally employed as an input parameter in the following P-BSHADE interpolation process.

\section{c. P-BSHADE interpolation models}

The objective of P-BSHADE is to interpolate the surface air temperature at the target location based on observed data from meteorological stations. The theoretical derivation of the formula is expressed as follows. The estimate of temperature $\hat{y}_{0}$ at the target location is expressed as

$$
\hat{y}_{0}=\sum_{i=1}^{n} w_{i} y_{i}
$$

where $w_{i}$ is the weight of the temperature recorder at the $i$ th station, and $y_{0}$ is the true but unknown temperature value to be estimated at a target location 0 .

The unbiasedness objective and the minimum estimation variance constraint regarding Eq. (1) are expressed as

$$
E\left(y_{0}\right)=E\left(\hat{y}_{0}\right) \text { and }
$$

$$
\min _{w}\left[\sigma_{\hat{y}_{0}}^{2}=E\left(\hat{y}_{0}-y_{0}\right)^{2}\right],
$$

where $E$ is the math expectation. Based on Eqs. (1) and (2), the relationship between the estimate at the target location and observation stations can be further expressed as

$$
E\left(y_{0}\right)=\sum_{i=1}^{n} w_{i} y_{i}
$$

In the real world, the field of Earth's surface air temperature is nonhomogeneous. In most cases, the temperatures at two locations are not equal, and the relationship can be further expressed as the relative bias $b_{i}$ between the mathematical expectation of $y_{0}$ and $y_{i}$. One of the important concept differentiating between P-BSHADE and the traditional geostatistical models is the introduction of the relative bias $b_{i}$ parameter, which gives this model the potential to remedy the biased sample:

$$
b_{i} E y_{0}=E y_{i} .
$$

By combining Eqs. (4) and (5), the relationship between the weight $w_{i}$ and the relative bias $b_{i}$ at station $i$ can be expressed as follows: 


$$
\sum_{i=1}^{n} w_{i} b_{i}=1
$$

The weight $w_{i}$ can be calculated using the unbiased condition constraint [Eq. (6)] and the objective function [Eq. (3)] is expressed as follows:

$$
\begin{aligned}
\sigma_{\hat{y}_{0}}^{2} & =\sigma_{y_{0}}^{2}+\sum_{i=1}^{n} \sum_{j=1}^{n} w_{i} w_{j} C\left(w_{i} w_{j}\right)-2 \sum_{i=1}^{n} w_{i} C\left(y_{i} y_{0}\right) \\
& +2 \mu\left(\sum_{i=1}^{n} w_{i} b_{i}-1\right),
\end{aligned}
$$

where $\mu$ is a Lagrange multiplier, and $C$ denotes statistical covariance between the temperature at two locations. Minimizing with respect to weights $w_{i}(i=1, \ldots, n)$, we obtain $w_{i}$ by solving the equation deduced by the first derivative of Eq. (7) with respect to $w$ and $\mu$.

Theoretically, the spatial distribution of land surface air temperature has the characteristics of spatial autocorrelation and heterogeneity, while stations will be biased if their distribution in the region is too sparse to represent all strata in the nonhomogeneous temperature field. In the P-BSHADE interpolation models, the characteristics of spatial autocorrelation of the temperature distribution can be depicted using the parameters of statistical covariance $C$ between two locations. In addition, the parameter relative bias $b_{i}$ at station $i$ was used to express the representativeness of a station and to remedy the bias of samples; it is also an indicator of the spatial heterogeneity. The theoretical advantage as well as parameterization and calculation process will result in lower error.

\section{Results}

\section{a. Interpolated grid temperature}

In this study, the combined spline with kriging (TPS$\mathrm{KRG}$ ) method was selected to produce the climatological field of the surface air temperature in the reference period because it has the smallest errors among the selected four methods. Detailed results are presented in the online supplemental material.

Figure 3 presents a map of the estimated surface air temperature spatial distribution using the P-BSHADE model for January, A pril, July, and October of 1905. The results for the same months in 1915, 1925, 1935, and 1945 are presented in Fig. S3 in the online supplemental material. A reasonable and detailed characterization is presented in these maps. It is difficult to represent the spatial variation of the surface air temperature in the regions of complex terrain by using commonly used methods; however, the spatial variation is clearly presented by the P-BSHADE model in the transition regions between different terrains, for example, at the edge of the Qinghai-Tibet Plateau, Tianshan Mountain in the westernmost part of China, and Hengduan Mountain in the southwest of China.

\section{b. Performance of P-BSHADE}

To assess the accuracy of the P-BSHADE method used in this study, two methods were used to perform validations. One of these methods was the leave-one-out cross-validation method using the real dataset in the historical period. In this process, each record was to be interpolated assuming that it does not exist. This method can be used to precisely reflect the estimation error at the locations of the observation stations using a real dataset for the historical period. Considering that in the historical period, the stations were all located in the eastern coastal areas of China, the estimation error in the vast middle and western region cannot be assessed. Therefore, another method was employed for simulating the station distribution in the historical period by using the dataset for recent years. The results of the two validation methods are described as follows.

\section{1) VALIDATION BY CROSS VALIDATION}

Results of the cross validation using the historical dataset from 1900 to 1950 show that P-BSHADE has smaller errors than those of the other methods. TPS-KRG is superior to kriging and IDW but has more errors than P-BSHADE; IDW yields the largest errors (Fig. 4). This implies that P-BSHADE is suitable for interpolation with lower uncertainty when the observation data are limited.

Before 1905, kriging and TPS-KRG did not work owing to the sparse distribution of stations, so only the results of these methods between 1905 and 1950 are summarized.

For each year from 1905 to 1950, the average RMSE for P-BSHADE is $0.98^{\circ} \mathrm{C}$, which is systematically smaller than the values obtained by the other three methods by $0.41^{\circ}-0.77^{\circ} \mathrm{C}$, the RMSE for TPS-KRG, kriging, and IDW are $1.46^{\circ}, 2.23^{\circ}$, and $2.64^{\circ} \mathrm{C}$, respectively.

The maximum RMSE of P-BSHADE is seen for December with a value of $1.38^{\circ} \mathrm{C}$, and the minimum RMSE is seen for August with a value of $0.8^{\circ} \mathrm{C}$. In contrast, the maximum RMSEs of TPS-KRG, kriging, and IDW are $1.84^{\circ}$ (in December), $2.45^{\circ}$ (in January), and $3.26^{\circ} \mathrm{C}$ (in January), respectively, and their minimum RMSEs are $1.15^{\circ}$ (in September), $1.96^{\circ}$ (in September), and $2.15^{\circ} \mathrm{C}$ (in August), respectively (Table 1). In each month, the RMSEs of P-BSHADE are all significantly lower than those from the other methods.

The index of MAE for the estimation error has similar features as the RMSE. P-BSHADE also shows the lowest MAE among the methods. For all months in the 

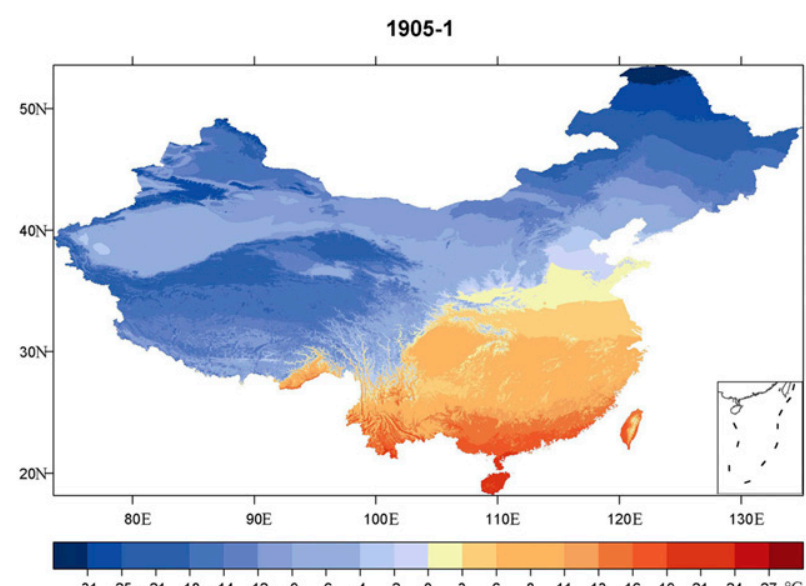

1905-7

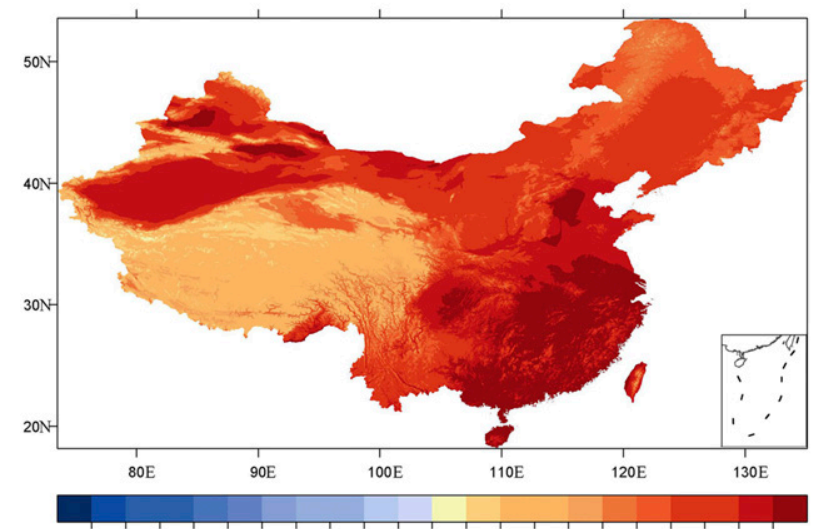

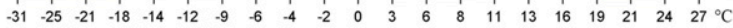

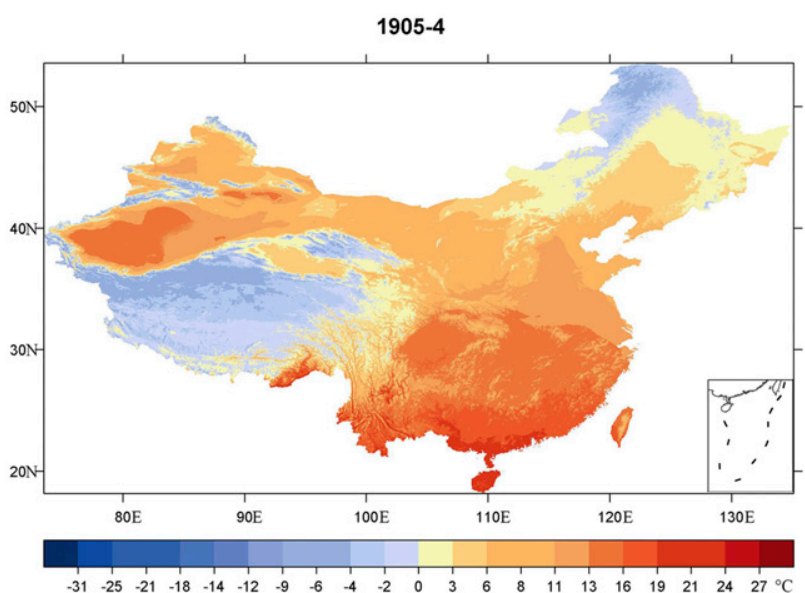

$\begin{array}{llllllllllllllllllllll}-31 & -25 & -21 & -18 & -14 & -12 & -9 & -6 & -4 & -2 & 0 & 3 & 6 & 8 & 11 & 13 & 16 & 19 & 21 & 24 & 27 & { }^{\circ} \mathrm{C}\end{array}$

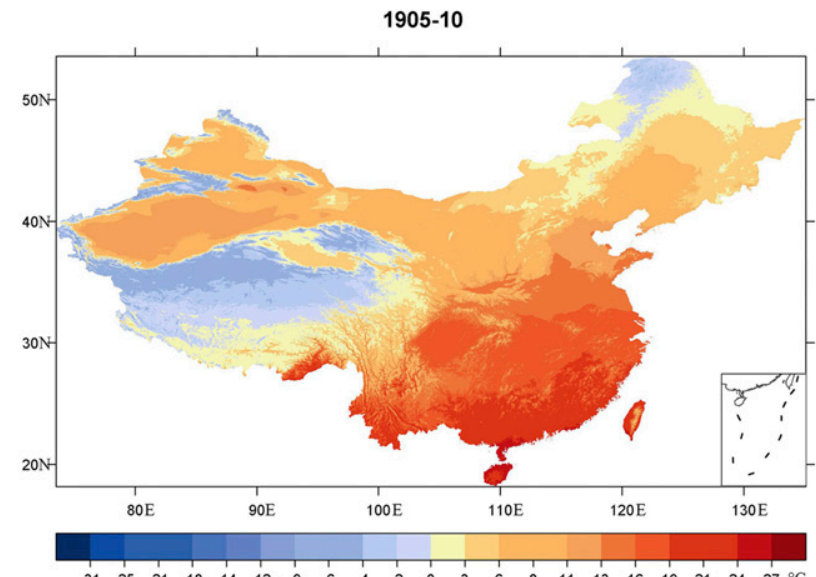

$\begin{array}{llllllllllllllllllllll}-31 & -25 & -21 & -18 & -14 & -12 & -9 & -6 & -4 & -2 & 0 & 3 & 6 & 8 & 11 & 13 & 16 & 19 & 21 & 24 & 27 & { }^{\circ} \mathrm{C}\end{array}$

FIG. 3. Map of estimated surface air temperature by P-BSHADE for (top left) January, (top right) April, (bottom left) July, and (bottom right) October 1905.

period of 1905-50, the average MAE for P-BSHADE is $0.75^{\circ} \mathrm{C}$, while the indexes for TPS-KRG, kriging, and IDW are $1.07^{\circ}, 1.51^{\circ}$, and $1.85^{\circ} \mathrm{C}$, respectively. Compared with P-BSHADE, the TPS-KRG, kriging, and IDW methods have significant differences between their RMSE and MAE, indicating that the results from these methods had large errors (Table 1).

The maximum MAE of P-BSHADE is seen for December with a value of $0.98^{\circ} \mathrm{C}$, and the minimum value is seen for May with a value of $0.61^{\circ} \mathrm{C}$. In contrast, the maximum MAEs of TPS-KRG, kriging and IDW are $1.36^{\circ}$ (in January), $1.69^{\circ}$ (in December), and $2.35^{\circ} \mathrm{C}$ (in January), respectively (Table 1 ). These values are also all significantly larger than that obtained from P-BSHADE.

\section{2) VALidATiOn By Simulating DATASET}

In this process, the validation in the period of 1961-90 was implemented. This process has the following steps.

First, a year in the early period (e.g., 1900) was selected, and the locations of stations were obtained.
Second, the corresponding station with the same location as that in the early years was used to predict the temperature at the locations of other stations in the period 1961-90. For each year during 1961-90, the stations were divided into two parts. One (labeled part 1) has the same locations as those in the early period (e.g., in 1900); these stations were used as observed values in the model. The other stations (labeled part 2) were used as locations to be interpolated. Third, for each year in the period 1961-90, the locations of part 2 were interpolated using the data from part 1 . Fourth, the RMSE and MAE for the cross validation in each year during 1961-90 were calculated. Finally, the average and standard deviation (SD) for both the RMSE and MAE for 1961-90 were calculated and used as the index to assess the performance of the interpolation in a year (e.g., 1900) in the early period (Fig. 5).

Similar to the results from the leave-one-out crossvalidation method using the real historical dataset, in the validation by simulating dataset, P-BSHADE exhibits 

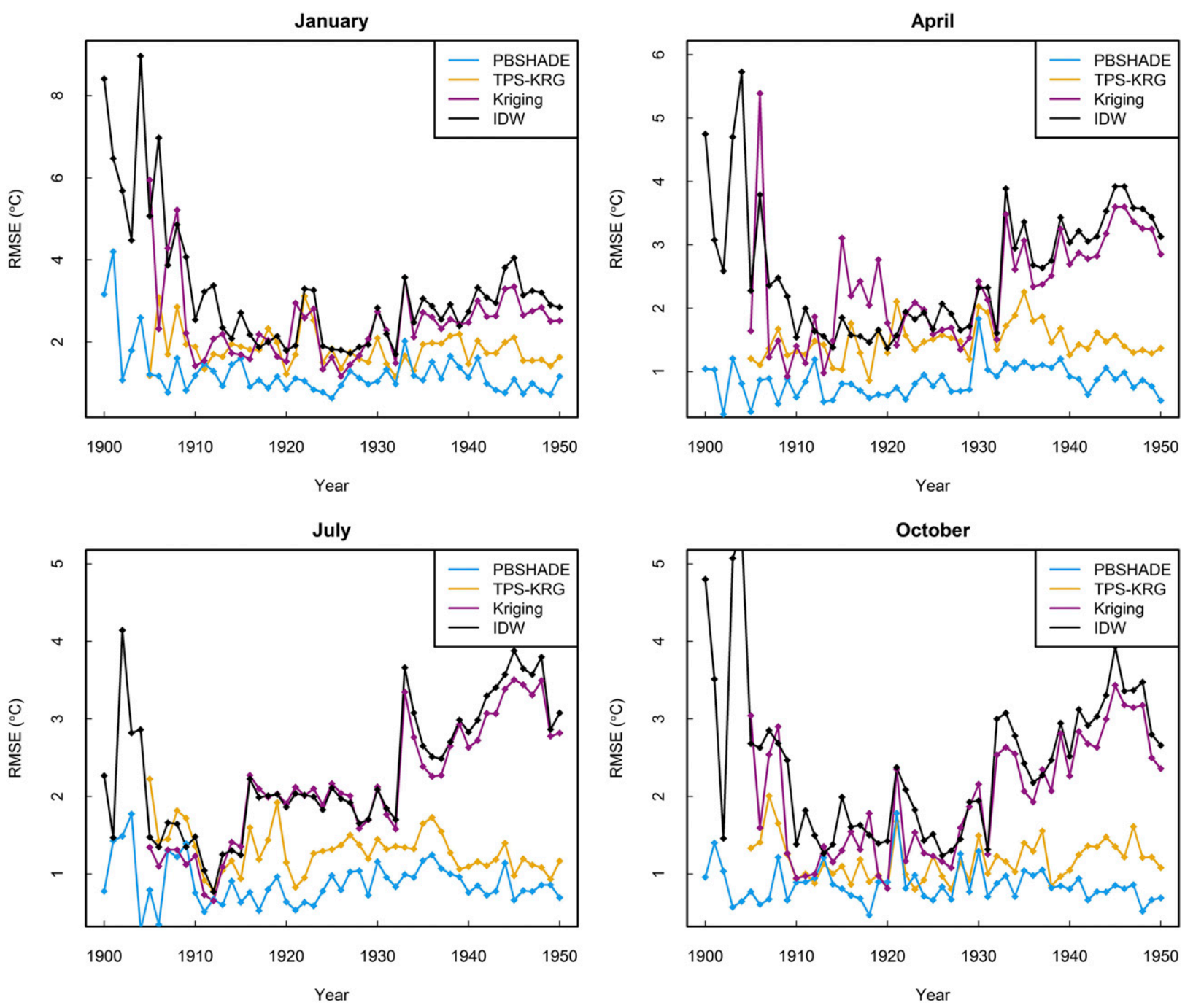

FIG. 4. Root-mean-square error (RMSE) in the period of 1900-50 estimated by leave-one-out cross validation: (top left) January, (top right) April, (bottom left) July, and (bottom right) October.

smaller errors than the other methods. Further, kriging and IDW have the largest errors, and TPS-KRG is superior to kriging and IDW but has more errors than P-BSHADE (Fig. 6).

The results show that the average RMSE for PBSHADE is $1.25^{\circ} \mathrm{C}$, while the indexes for TPS-KRG, kriging, and IDW are $5.40^{\circ}, 5.34^{\circ}$, and $5.34^{\circ} \mathrm{C}$, respectively. P-BSHADE systematically has smaller estimated errors than the other three methods by $4.09^{\circ}-4.78^{\circ} \mathrm{C}$.

The maximum RMSE of P-BSHADE is seen for January with a value of $1.74^{\circ} \mathrm{C}$, and the minimum RMSE is seen for September with a value of $0.98^{\circ} \mathrm{C}$. Furthermore, the estimated errors are stable as the standard deviation of RMSE range is small from $0.03^{\circ}$ to $0.11^{\circ} \mathrm{C}$ over 12 months. Compared with P-BSHADE, the other three methods present larger errors in each month. For example, the maximum RMSEs of TPS-KRG, kriging, and IDW are $7.61^{\circ} \mathrm{C}$ (in January), $7.02^{\circ} \mathrm{C}$ (in March), and $5.91^{\circ} \mathrm{C}$ (in January), respectively, while their minimum RMSEs are $4.07^{\circ} \mathrm{C}$ (in October), $4.99^{\circ} \mathrm{C}$ (in June), and $4.99^{\circ} \mathrm{C}$ (in July), respectively. The RMSEs of the other methods are all significantly larger than the RMSE of P-BSHADE in the same month (Table 2).

The estimated error is influenced by the sparsity of stations, although compared with the other three methods, P-BSHADE is not sensible. The estimated error in the early years with sparse stations does not appear apparently larger than that in the years having relatively dense stations. For example, for the 10 years between 1905 and 1914 in January, the average RMSE for P-BSHADE is $1.8^{\circ} \mathrm{C}$, whereas for the 10 years 
TABLE 1. RMSE/MAE in each month in the period of 1905-50 estimated by the leave-one-out cross validation procedure $\left({ }^{\circ} \mathrm{C}\right)$

\begin{tabular}{cccll}
\hline \hline Month & P-BSHADE & TPS-KRG & \multicolumn{1}{c}{ Kriging } & \multicolumn{1}{c}{ IDW } \\
\hline 1 & $1.25 / 0.96$ & $1.82 / 1.36$ & $2.45 / 1.69$ & $3.26 / 2.35$ \\
2 & $1.15 / 0.87$ & $1.79 / 1.31$ & $2.22 / 1.57$ & $2.97 / 2.14$ \\
3 & $0.99 / 0.75$ & $1.63 / 1.12$ & $2.14 / 1.46$ & $2.69 / 1.91$ \\
4 & $0.85 / 0.65$ & $1.49 / 1.05$ & $2.33 / 1.53$ & $2.62 / 1.84$ \\
5 & $0.8 / 0.61$ & $1.34 / 1.01$ & $2.41 / 1.6$ & $2.57 / 1.86$ \\
6 & $0.91 / 0.7$ & $1.37 / 1.01$ & $2.28 / 1.54$ & $2.49 / 1.77$ \\
7 & $0.88 / 0.7$ & $1.3 / 0.96$ & $2.15 / 1.42$ & $2.32 / 1.6$ \\
8 & $0.8 / 0.63$ & $1.15 / 0.84$ & $2.15 / 1.38$ & $2.15 / 1.43$ \\
9 & $0.82 / 0.64$ & $1.15 / 0.87$ & $1.96 / 1.33$ & $2.17 / 1.47$ \\
10 & $0.86 / 0.66$ & $1.18 / 0.89$ & $1.98 / 1.35$ & $2.44 / 1.69$ \\
11 & $1.05 / 0.79$ & $1.4 / 1.03$ & $2.2 / 1.5$ & $2.81 / 1.97$ \\
12 & $1.38 / 0.98$ & $1.84 / 1.33$ & $2.43 / 1.69$ & $3.18 / 2.21$ \\
\hline
\end{tabular}

between 1941 and 1950 in January the average RMSE for P-BSHADE is $1.67^{\circ} \mathrm{C}$. The other methods appeared to have significant differences in performance for different station densities; they present comparable differences in the estimated errors. For example, in the 10 years between 1905 and 1914 in January, the average RMSEs for TPS-KRG, kriging, and IDW are $24.77^{\circ}$, $7.53^{\circ}$, and $6.24^{\circ} \mathrm{C}$, respectively, whereas for the 10 years between 1941 and 1950 in January the average RMSEs for the three methods are $2.54^{\circ}, 4.67^{\circ}$, and $5.06^{\circ} \mathrm{C}$, respectively.

The index of MAE for the estimation error shows that P-BSHADE has lowest MAE compared to the other methods. For all months, the average mean MAE for P-BSHADE is $0.85^{\circ} \mathrm{C}$, while the indexes for TPS-KRG, kriging, and IDW are $3.51^{\circ}, 3.77^{\circ}$, and $3.56^{\circ} \mathrm{C}$, respectively (Table 3 ).

The maximum MAE of P-BSHADE is seen for January with a value of $1.13^{\circ} \mathrm{C}$, and the minimum value $\left(0.69^{\circ} \mathrm{C}\right)$ is seen for September. The other three methods present larger MAEs. For example, the maximum MAE values of TPS-KRG, kriging, and IDW are $5.06^{\circ}, 4.09^{\circ}$, and $4.11^{\circ} \mathrm{C}$, respectively. Besides exhibiting small interpolation errors, P-BSHADE also presents a stable level; for example, the average standard deviation of the MAE over 12 months is $0.052^{\circ} \mathrm{C}$ for P-BSHADE, while the values for the other methods range from $0.92^{\circ}$ to $4.99^{\circ} \mathrm{C}$ (Table 3).

P-BSHADE presents the trend that the MAEs decrease as the density increases from 1905 to 1950 . The other methods also present the same characteristic, with higher values and obvious variations. For example, the
Stations in historical year (e.g.1900)

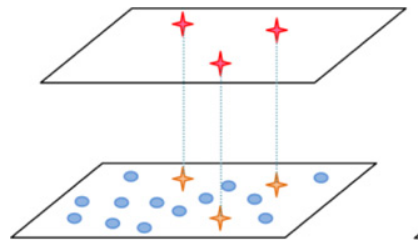

Stations in reference period (e.g.1961)
Stations in historical year

(e.g.1900)

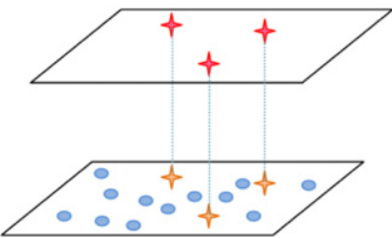

Stations in reference period

(e.g.1962)
Stations in historical year (e.g.1900)

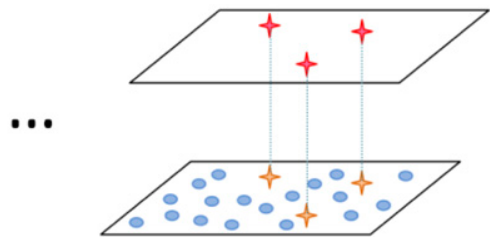

Stations in reference period

(e.g.1990)

Legend

$\downarrow$ : Station in historical years.

$\downarrow$ : Station in reference period years (part 1), which has the same location as a station in early year, used as predictor in simulation of historical situation in validation calculation.

Station in reference period year (part 2), which is assumed to not exist and then to be predicted in the validation calculation.

FIG. 5. Diagram of the cross validation of the historical period temperature interpolation using stations in recent years. 

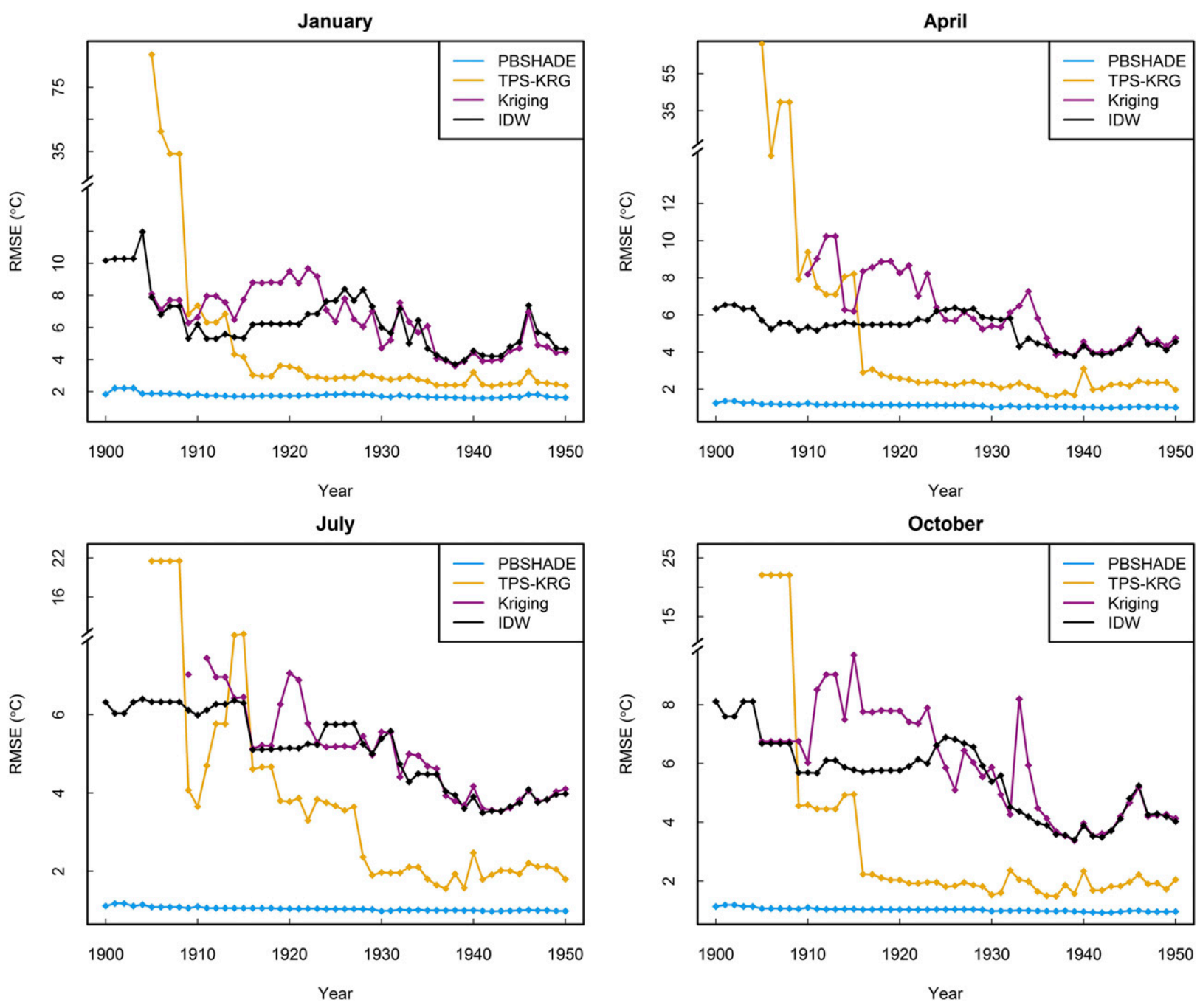

FIG. 6. Root-mean-square error (RMSE) in the period of 1961-90 estimated by simulating the dataset using only stations that existed between 1900 and 1950: (top left) January, (top right) April, (bottom left) July, and (bottom right) October.

MAE for P-BSHADE is $1.21^{\circ} \mathrm{C}$ between 1905 and 1914 in January, while the values for TPS-KRG, kriging, and IDW are $16.16^{\circ}, 4.94^{\circ}$, and $4.48^{\circ} \mathrm{C}$, respectively. Meanwhile, the MAE for P-BSHADE is $1.07^{\circ} \mathrm{C}$ between 1941 and 1950 in January, and the values for TPS-KRG, kriging, and IDW are $1.73^{\circ}, 3.20^{\circ}$, and $3.52^{\circ} \mathrm{C}$, respectively.

\section{c. Mapping the uncertainty of the interpolated results}

The P-BSHADE method has the advantage of uncertainty assessment for the estimated results, and the uncertainty map corresponding to the interpolated temperature can be produced by the index of estimated error variance using Eq. (7). The maps are shown in Figs. 7 and S4, in which the results for the years 1905, 1915, 1925, 1935, and 1945 are presented, reflecting the influence of different station densities. In each year, four months-January, April, July, and October-were selected in order to reveal the influence of seasons.

The uncertainty of the estimated results was larger in the early years (e.g., in 1905), when the stations were sparse and spatially unevenly distributed, and the uncertainty decreased as the number of stations increased in later years (e.g., in 1945). This phenomenon was observed for all seasons. This implies that the sparse and uneven spatial distribution of stations is an important source of uncertainty.

In different seasons, there is an apparent difference in the uncertainty. The uncertainty in winter is larger than that in summer and autumn. This is consistent with the results in the cross validation using the dataset in the reference period; for example, in winter, the average MAE of P-BSHADE is $1.12^{\circ} \mathrm{C}$, whereas in summer the value is $0.71^{\circ} \mathrm{C}$. 
TABLE 2. RMSE and its corresponding standard error (SD) in each month for different methods $\left({ }^{\circ} \mathrm{C}\right)$

\begin{tabular}{|c|c|c|c|c|}
\hline & P-BSHADE & TPS-KRG & Kriging & IDW \\
\hline Month & RMSE (SD of RMSE) & RMSE (SD of RMSE) & RMSE (SD of RMSE) & RMSE (SD of RMSE) \\
\hline 1 & $1.74(0.08)$ & $7.61(15.96)$ & $6.45(1.81)$ & $5.91(1.28)$ \\
\hline 2 & $1.73(0.11)$ & $6.20(8.11)$ & $6.82(1.77)$ & $5.67(1.04)$ \\
\hline 3 & $1.38(0.07)$ & $6.20(8.48)$ & $7.02(2.53)$ & $5.34(0.80)$ \\
\hline 4 & $1.10(0.06)$ & $6.53(12.57)$ & $6.19(1.89)$ & $5.12(0.77)$ \\
\hline 5 & $1.05(0.05)$ & $5.83(9.04)$ & $5.31(1.00)$ & $5.09(0.79)$ \\
\hline 6 & $1.03(0.04)$ & $5.79(8.87)$ & $4.99(0.83)$ & $5.02(0.83)$ \\
\hline 7 & $1.03(0.03)$ & $4.93(5.98)$ & $5.06(1.16)$ & $4.99(0.97)$ \\
\hline 8 & $1.01(0.03)$ & $4.37(4.34)$ & $5.72(1.82)$ & $5.07(1.06)$ \\
\hline 9 & $0.98(0.04)$ & $4.27(5.58)$ & $5.79(1.72)$ & $5.02(1.07)$ \\
\hline 10 & $1.01(0.04)$ & $4.07(5.71)$ & $5.98(1.77)$ & $5.26(1.12)$ \\
\hline 11 & $1.27(0.07)$ & $4.30(5.10)$ & $6.58(2.09)$ & $5.75(1.22)$ \\
\hline 12 & $1.67(0.11)$ & $4.72(5.56)$ & $6.44(2.02)$ & $5.88(1.30)$ \\
\hline
\end{tabular}

When comparing the uncertainty in different years, a higher uncertainty is observed in the area of the QinghaiTibet Plateau and northwest China. This area is covered by large-scale mountains, which influences the spatial distribution of the surface air temperature. This implies that a large-scale mountain area is another important source of uncertainty in the temperature mapping.

\section{Conclusions and discussion}

In this study, the P-BSHADE method has been used to interpolate the spatial distribution of monthly surface air temperatures in the historical period of 1900-50. The results of P-BSHADE have been compared with those of other widely used methods, such as kriging, IDW, and a combination of spline with kriging. The findings suggest that the P-BSHADE method has low uncertainty, owing to its theoretical advantages, while cross validation also indicated that it has the smallest estimated error and demonstrated its empirical superiority.

Compared with other traditional methods, the P-BSHADE model has an advantage in interpolation of temperature using stations in the historical period. P-BSHADE considered important factors influencing temperature interpolation in the historical period, including the issue of spatial representation of sparse and unevenly spatially distributed stations, and the spatial relationship from surface air temperature fields (Jones et al. 1999; New et al. 1999, 2000).

Compared with the missing data estimation for existing stations, grid interpolation is more challenging, because the issues of quantification the characteristics of spatial relationships for the temperature field and the relative bias for station distribution should be solved. This study provides a solution for the parameterization and calculation process for grid interpolation using $\mathrm{P}$ BSHADE.

TABLE 3. MAE and its corresponding standard error (SD) in each month for different methods $\left({ }^{\circ} \mathrm{C}\right)$

\begin{tabular}{|c|c|c|c|c|}
\hline & P-BSHADE & TPS-KRG & Kriging & IDW \\
\hline Month & $\overline{\text { MAE (SD of MAE) }}$ & $\overline{\text { MAE (SD of MAE) }}$ & $\overline{\text { MAE (SD of MAE) }}$ & MAE (SD of MAE) \\
\hline 1 & $1.13(0.07)$ & $5.06(10.45)$ & $4.09(1.01)$ & $4.11(0.91)$ \\
\hline 2 & $1.12(0.08)$ & $4.07(5.21)$ & $4.23(0.95)$ & $3.90(0.74)$ \\
\hline 3 & $0.94(0.06)$ & $4.01(5.28)$ & $4.16(1.15)$ & $3.58(0.54)$ \\
\hline 4 & $0.78(0.05)$ & $4.23(7.86)$ & $3.88(1.05)$ & $3.36(0.47)$ \\
\hline 5 & $0.75(0.04)$ & $3.73(5.58)$ & $3.42(0.61)$ & $3.27(0.46)$ \\
\hline 6 & $0.72(0.04)$ & $3.65(5.48)$ & $3.22(0.48)$ & $3.20(0.49)$ \\
\hline 7 & $0.71(0.03)$ & $3.17(3.75)$ & $3.23(0.69)$ & $3.17(0.59)$ \\
\hline 8 & $0.70(0.04)$ & $2.87(2.79)$ & $3.54(1.02)$ & $3.24(0.68)$ \\
\hline 9 & $0.69(0.03)$ & $2.75(3.43)$ & $3.65(0.98)$ & $3.33(0.74)$ \\
\hline 10 & $0.71(0.03)$ & $2.65(3.52)$ & $3.75(0.95)$ & $3.55(0.79)$ \\
\hline 11 & $0.87(0.06)$ & $2.84(3.17)$ & $4.04(1.03)$ & $3.92(0.87)$ \\
\hline 12 & $1.10(0.08)$ & $3.14(3.44)$ & $4.05(1.10)$ & $4.09(0.95)$ \\
\hline
\end{tabular}


$1905-1$
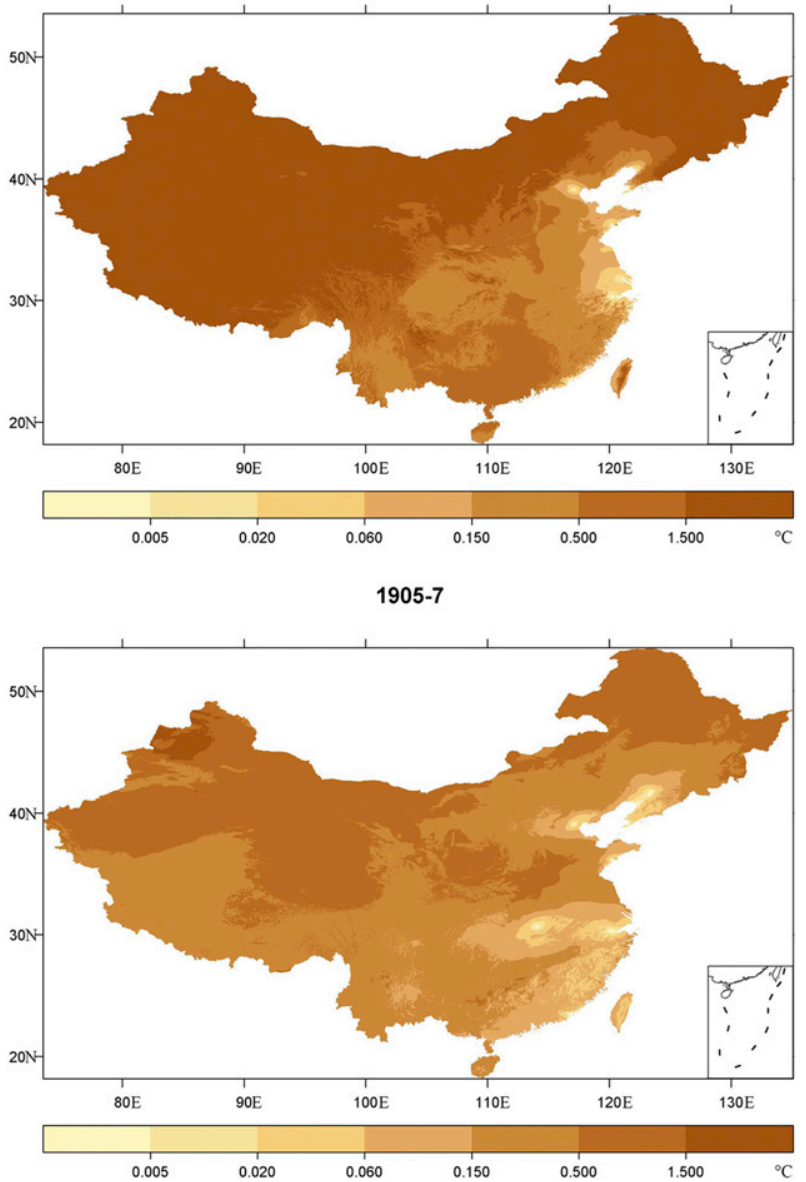

$1905-4$

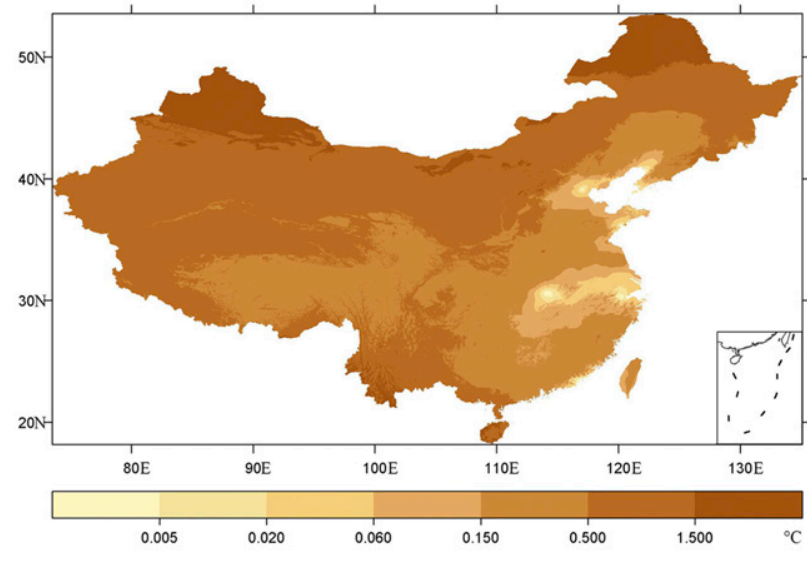

$1905-10$

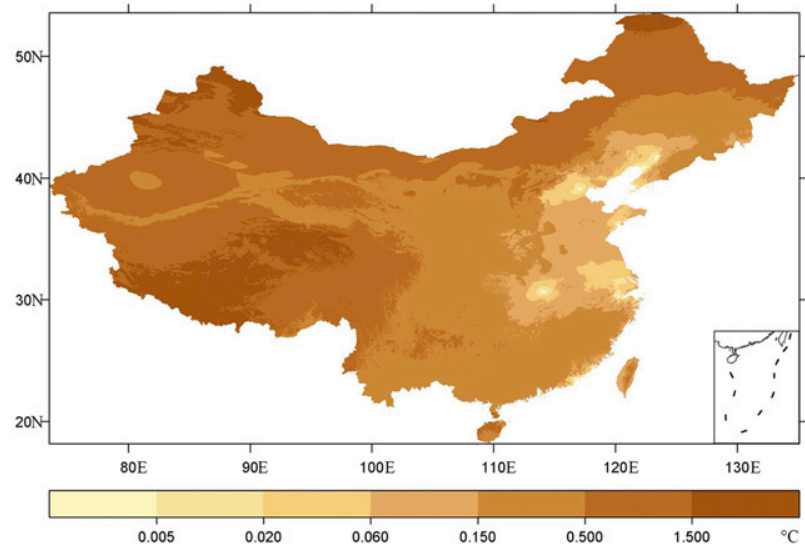

FIG. 7. Map of surface air temperature estimated error variance by P-BSHADE for 1905 in (top left) January, (top right) April, (bottom left) July, and (bottom right) October.

The phenomena of spatial autocorrelation and spatial heterogeneity in land surface temperatures are two important characteristics of spatial relationships that are typical in geographic objects. The surface air temperatures of nearby locations have a greater correlation owing to their similar geographic location and land surface characteristics influencing the temperature spatial distribution, which is the phenomenon of spatial autocorrelation. The surface air temperature is affected by typical factors, such as elevation, slope, and aspect (Daly et al. 2002; Karl and Jones 1989). In some cases, two locations that are far apart will have a higher correlation than those in closer proximity owing to their similar geography. This is the phenomenon of heterogeneity of temperature distributions (Böhm et al. 2001). Compared with P-BSHADE, other methods (e.g., the widely used geostatistical method or IDW) assume a second-order stationary process, or only consider the spatial relationship of homogeneity. Conversely, in P-BSHADE, the covariance between two locations is calculated by the time series, which avoids the constraint of assumptions of the geostatistical method.

The sparse and unevenly spatially distributed stations in early years (e.g., in the 1900s) triggered the issue of the spatial representation of observations. A sample will be biased if its size is too small to cover all strata in a heterogeneous surface (Wang et al. 2012). Compared to existing methods, the P-BSHADE model can remedy a biased sample, thus leading to a lower estimation error (Figs. 4 and 6; see also Fig. S5). In the P-BSHADE model, the problem is solved by introducing an index of the relative ratio [ $b$ value in Eq. (5)] between stations and the location to be interpolated. It is also an indicator of the spatial heterogeneity of the temperature distributions. For example, in the early 1900s, there were only a few stations, and they were all distributed in the most eastern part of China, so their locations cannot represent the temperature throughout the entire country, especially in the northwest and Qinghai-Tibet Plateau of China. The results estimated by P-BSHADE 
have smaller errors and are robust, owing to its potential to remedy the biased sample.

Besides the sampling error, terrain is another important factor causing diversity in the spatial distribution of surface air temperatures, and it is challenging for climate interpolation. In this study, the DEM data were used to reflect the effect of terrain. Although the spatial distribution of stations is sparse in the historical period, the information of the terrain effect in the reference period can be used in P-BSHADE, so that the elevation-related details can be well represented in the interpolated map. However, local influence is also present in the estimation in local regions. Higher errors from P-BSHADE were mainly located in western China. Figure S6 shows the top 100 errors in the cross validation using the dataset for January, April, July, and October of 1975, in which only the stations at the same locations in 1910, 1920, 1930, 1940, and 1950 were selected as predictors in the interpolation. The results show that the stations with the highest errors were mainly located in the inner area and the eastern edge of the Qinghai-Tibet Plateau, around the Tianshan Mountains in western China, because of the complex terrain and sparse stations in that region.

There are some sources of uncertainties related to the estimation. On one hand, uncertainty is a result of the parameters in some regions. In the P-BSHADE model, the spatial relationships were extracted from the observation values in the stations, and the spatial arrangement of the stations is reflective of human knowledge and the observation capabilities, which influences the calculation of spatial relationship for surface air temperature. On the other hand, uncertainty comes from the dataset. The data employed in the study have been homogenized and quality controlled to reduce evidently large errors ( $\mathrm{Li}$ et al. 2010, 2017; W. Xu et al. 2013; Yan and Jones 2008); however, this does not fully eliminate the influence of urbanization, the change of instruments or the local environment, and the movement of the stations (Yan et al. 2010), especially in the early years. In addition, the difference of the homogenization and quality control process will also introduce uncertainty.

This method is not limited to the interpolation of surface air temperatures, and it can be further applied to other climate factors (e.g., precipitation) as it is a common method. The only difference in the calculation process is the parameter. Meanwhile, sparse stations were observed not only during the historical period, but also in recent years in some areas, such as Antarctica. Furthermore, climate data are interrelated both in space and time, and this information is most useful since it allows the reconstruction of the space-time state of the atmosphere from a limited number of observations characterized by uncertainties from various sources (soft data) (Christakos 1992; Yu et al. 2010). In future studies, the parameters of cross-space-time dependencies will be quantified in the frame of P-BSHADE by incorporating soft data.

Acknowledgments. This study was funded by the Ministry of Science and Technology of China (GYHY20140616) and the National Science Foundation of China $(41531179,41601419)$.

\section{REFERENCES}

Boer, E. P. J., K. M. de Beurs, and A. D. Hartkamp, 2001: Kriging and thin plate splines for mapping climate variables. Int. J. Appl. Earth Obs. Geoinf., 3, 146-154, https://doi.org/ 10.1016/S0303-2434(01)85006-6.

Böhm, R., I. Auer, M. Brunetti, M. Maugeri, T. Nanni, and W. Schöner, 2001: Regional temperature variability in the European Alps: 1760-1998 from homogenized instrumental time series. Int. J. Climatol., 21, 1779-1801, https://doi.org/ 10.1002/joc.689.

Brohan, P., J. J. Kennedy, I. Harris, S. F. B. Tett, and P. D. Jones, 2006: Uncertainty estimates in regional and global observed temperature changes: A new data set from 1850. J. Geophys. Res., 111, D12106, https://doi.org/10.1029/2005JD006548.

Burrough, P. A., and R. A. McDonnell, 1998: Principles of Geographical Information Systems. Oxford University Press, 333 pp.

Cao, L. J., P. Zhao, Z. W. Yan, P. Jones, Y. N. Zhu, Y. Yu, and G. L. Tang, 2013: Instrumental temperature series in eastern and central China back to the nineteenth century. J. Geophys. Res. Atmos., 118, 8197-8207, https://doi.org/10.1002/jgrd.50615.

_, Y. N. Zhu, G. L. Tang, F. Yuan, and Z. W. Yan, 2016: Climatic warming in China according to a homogenized data set from 2419 stations. Int. J. Climatol., 36, 4384-4392, https:// doi.org/10.1002/joc.4639.

- Z. W. Yan, P. Zhao, Y. N. Zhu, Y. Yu, G. L. Tang, and P. Jones, 2017: Climatic warming in China during 1901-2015 based on an extended dataset of instrumental temperature records. Environ. Res. Lett., 12, 064005, https://doi.org/ 10.1088/1748-9326/aa68e8.

Christakos, G., 1992: Random Field Models in Earth Sciences. Academic Press, 474 pp.

Daly, C., 2006: Guidelines for assessing the suitability of spatial climate data sets. Int. J. Climatol., 26, 707-721, https://doi.org/ 10.1002/joc.1322.

- W. P. Gibson, G. H. Taylor, G. L. Johnson, and P. Pasteris, 2002: A knowledge-based approach to the statistical mapping of climate. Climate Res., 22, 99-113, https://doi.org/10.3354/ cr022099.

— E. H. Helmer, and M. Quiñones, 2003: Mapping the climate of Puerto Rico, Vieques and Culebra. Int. J. Climatol., 23, 1359-1381, https://doi.org/10.1002/joc.937.

DeGaetano, A. T., and B. N. Belcher, 2007: Spatial interpolation of daily maximum and minimum air temperature based on meteorological model analyses and independent observations. J. Appl. Meteor. Climatol., 46, 1981-1992, https://doi.org/ 10.1175/2007JAMC1536.1.

Di Luzio, M., G. L. Johnson, C. Daly, J. K. Eischeid, and J. G. Arnold, 2008: Constructing retrospective gridded daily precipitation and temperature datasets for the conterminous 
United States. J. Appl. Meteor. Climatol., 47, 475-497, https:// doi.org/10.1175/2007JAMC1356.1.

Di Piazza, A., F. Lo Conti, L. V. Noto, F. Viola, and G. La Loggia, 2011: Comparative analysis of different techniques for spatial interpolation of rainfall data to create a serially complete monthly time series of precipitation for Sicily, Italy. Int. J. Appl. Earth Obs. Geoinf., 13, 396-408, https://doi.org/ 10.1016/j.jag.2011.01.005.

Eischeid, J. K., P. A. Pasteris, H. F. Diaz, M. S. Plantico, and N. J. Lott, 2000: Creating a serially complete, national daily time series of temperature and precipitation for the western United States. J. Appl. Meteor., 39, 1580-1591, https://doi.org/ 10.1175/1520-0450(2000)039<1580:CASCND>2.0.CO;2.

Fan, Y., and H. van den Dool, 2008: A global monthly land surface air temperature analysis for 1948-present. J. Geophys. Res., 113, D01103, https://doi.org/10.1029/2007JD008470.

Goovaerts, P., 1997: Geostatistics for Natural Resources Evaluation. Oxford University Press, 483 pp.

Guan, H., X. P. Zhang, O. Makhnin, and Z. A. Sun, 2013: Mapping mean monthly temperatures over a coastal hilly area incorporating terrain aspect effects. J. Hydrometeor., 14, 233250, https://doi.org/10.1175/JHM-D-12-014.1.

Haining, R., 2003: Spatial Data Analysis: Theory and Practice. Cambridge University Press, 432 pp.

Hansen, J., and S. Lebedeff, 1987: Global trends of measured surface air temperature. J. Geophys. Res., 92, 13 345-13 372, https://doi.org/10.1029/JD092iD11p13345.

—, R. Ruedy, M. Sato, and K. Lo, 2010: Global surface temperature change. Rev. Geophys., 48, RG4004, https://doi.org/ 10.1029/2010RG000345.

Hartmann, D. L., and Coauthors, 2013: Observations: Atmosphere and surface. Climate Change 2013: The Physical Science Basis, T. F. Stocker et al., Eds., Cambridge University Press, 159-254.

Haylock, M. R., N. Hofstra, A. M. G. Klein Tank, E. J. Klok, P. D. Jones, and M. New, 2008: A European daily highresolution gridded data set of surface temperature and precipitation for 1950-2006. J. Geophys. Res., 113, D20119, https://doi.org/10.1029/2008JD010201.

Hijmans, R. J., S. E. Cameron, J. L. Parra, P. G. Jones, and A. Jarvis, 2005: Very high resolution interpolated climate surfaces for global land areas. Int. J. Climatol., 25, 1965-1978, https://doi.org/10.1002/joc.1276.

Hofstra, N., M. Haylock, M. New, P. Jones, and C. Frei, 2008: Comparison of six methods for the interpolation of daily, European climate data. J. Geophys. Res., 113, D21110, https:// doi.org/10.1029/2008JD010100.

Hudson, G., and H. Wackernagel, 1994: Mapping temperature using kriging with external drift: Theory and an example from Scotland. Int. J. Climatol., 14, 77-91, https://doi.org/10.1002/ joc.3370140107.

Isaaks, E. H., and R. M. Srivastava, 1989: Applied Geostatistics. Oxford University Press, $561 \mathrm{pp}$.

Jeffrey, S. J., J. O. Carter, K. B. Moodie, and A. R. Beswick, 2001: Using spatial interpolation to construct a comprehensive archive of Australian climate data. Environ. Modell. Software, 16, 309-330, https://doi.org/10.1016/S1364-8152(01)00008-1.

Jones, P. D., 1994: Hemispheric surface air temperature variations: A reanalysis and an update to 1993. J. Climate, 7, 1794-1802, https://doi.org/10.1175/1520-0442(1994)007<1794: HSATVA $>2.0 . \mathrm{CO} ; 2$.

__ 2016: The reliability of global and hemispheric surface temperature records. Adv. Atmos. Sci., 33, 269-282, https:// doi.org/10.1007/s00376-015-5194-4.
— and D. H. Lister, 2009: The urban heat island in central London and urban-related warming trends in central London since 1900. Weather, 64, 323-327, https://doi.org/10.1002/wea.432.

— , and T. M. L. Wigley, 2010: Estimation of global temperature trends: What's important and what isn't. Climatic Change, 100, 59-69, https://doi.org/10.1007/s10584-010-9836-3.

—, M. New, D. E. Parker, S. Martin, and I. G. Rigor, 1999: Surface air temperature and its changes over the past 150 years. Rev. Geophys., 37, 173-199, https://doi.org/10.1029/ 1999RG900002.

, D. H. Lister, T. J. Osborn, C. Harpham, M. Salmon, and C. P. Morice, 2012: Hemispheric and large-scale land-surface air temperature variations: An extensive revision and an update to 2010. J. Geophys. Res., 117, D05127, https://doi.org/ 10.1029/2011JD017139.

Karl, T. R., and P. D. Jones, 1989: Urban bias in area-averaged surface air temperature trends. Bull. Amer. Meteor. Soc., 70, 265-270, https://doi.org/10.1175/1520-0477(1989)070<0265: UBIAAS $>2.0 . \mathrm{CO} ; 2$.

Kilibarda, M., T. Hengl, G. B. M. Heuvelink, B. Gräler, E. Pebesma, M. Perčec Tadić, and B. Bajat, 2014: Spatiotemporal interpolation of daily temperatures for global land areas at $1 \mathrm{~km}$ resolution. J. Geophys. Res. Atmos., 119, 22942313, https://doi.org/10.1002/2013JD020803.

Lawrimore, J. H., M. J. Menne, B. E. Gleason, C. N. Williams, D. B. Wuertz, R. S. Vose, and J. Rennie, 2011: An overview of the Global Historical Climatology Network monthly mean temperature data set, version 3. J. Geophys. Res. Atmos., 116, D19121, https://doi.org/10.1029/2011JD016187.

Li, Q., W. Dong, W. Li, X. Gao, P. Jones, J. Kennedy, and D. Parker, 2010: Assessment of the uncertainties in temperature change in China during the last century. Chin. Sci. Bull., 55, 1974-1982, https://doi.org/10.1007/s11434-010-3209-1.

—, L. Zhang, W. Xu, T. Zhou, J. Wang, P. Zhai, and P. Jones, 2017: Comparisons of time series of annual mean surface air temperature for China since the 1900s. Bull. Amer. Meteor. Soc., 98, 699-711, https://doi.org/10.1175/BAMS-D-16-0092.1.

Mitchell, T. D., and P. D. Jones, 2005: An improved method of constructing a database of monthly climate observations and associated high-resolution grids. Int. J. Climatol., 25, 693-712, https://doi.org/10.1002/joc.1181.

Nalder, I. A., and R. W. Wein, 1998: Spatial interpolation of climatic normals: Test of a new method in the Canadian boreal forest. Agric. For. Meteor., 92, 211-225, https://doi.org/ 10.1016/S0168-1923(98)00102-6.

New, M., M. Hulme, and P. Jones, 1999: Representing twentiethcentury space-time climate variability. Part I: Development of a 1961-90 mean monthly terrestrial climatology. J. Climate, 12, 829-856, https://doi.org/10.1175/1520-0442(1999)012<0829: RTCSTC $>2.0 . C O ; 2$.

,-- , and,- 2000 : Representing twentieth-century spacetime climate variability. Part II: Development of 190196 monthly grids of terrestrial surface climate. J. Climate, 13, 2217-2238, https://doi.org/10.1175/1520-0442(2000)013<2217: RTCSTC $>2.0 . \mathrm{CO} ; 2$.

Olea, R. A., 1999: Geostatistics for Engineers and Earth Scientists. Springer, $303 \mathrm{pp}$.

Piper, S. C., and E. F. Stewart, 1996: A gridded global data set of daily temperature and precipitation for terrestrial biospheric modeling. Global Biogeochem. Cycles, 10, 757-782, https:// doi.org/10.1029/96GB01894.

Stahl, K., R. D. Moore, J. A. Floyer, M. G. Asplin, and I. G. McKendry, 2006: Comparison of approaches for spatial 
interpolation of daily air temperature in a large region with complex topography and highly variable station density. Agric. For. Meteor., 139, 224-236, https://doi.org/10.1016/ j.agrformet.2006.07.004.

Wang, J.-F., and Coauthors, 2011: Area disease estimation based on sentinel hospital records. PLoS One, 6, e23428, https://doi.org/ 10.1371/journal.pone.0023428.

_- A. Stein, B.-B. Gao, and Y. Ge, 2012: A review of spatial sampling. Spat. Stat., 2, 1-14, https://doi.org/10.1016/ j.spasta.2012.08.001.

, C.-D. Xu, M.-G. Hu, Q.-X. Li, Z.-W. Yan, P. Zhao, and P. Jones, 2014: A new estimate of the China temperature anomaly series and uncertainty assessment in 1900-2006. J. Geophys. Res. Atmos., 119, 1-9, https://doi.org/10.1002/ 2013JD020542.

Xu, C.-D., J.-F. Wang, M.-G. Hu, and Q.-X. Li, 2013: Interpolation of missing temperature data at meteorological stations using P-BSHADE. J. Climate, 26, 7452-7463, https://doi.org/ 10.1175/JCLI-D-12-00633.1.
Xu, W., Q. Li, X. L. Wang, S. Yang, L. Cao, and Y. Feng, 2013: Homogenization of Chinese daily surface air temperatures and analysis of trends in the extreme temperature indices. J. Geophys. Res. Atmos., 118, 9708-9720, https://doi.org/ 10.1002/jgrd.50791.

_ , and Coauthors, 2017: A new integrated and homogenized global monthly land surface air temperature dataset for the period since 1900. Climate Dyn., https://doi.org/10.1007/ s00382-017-3755-1.

Yan, Z., and P. Jones, 2008: Detecting inhomogeneity in daily climate series using wavelet analysis. Adv. Atmos. Sci., 25, 157163, https://doi.org/10.1007/s00376-008-0157-7.

_ Z. Zi, Q. Li, and P. Jones, 2010: Effects of site change and urbanisation in the Beijing temperature series 1977-2006. Int J. Climatol., 30, 1226-1234, https://doi.org/10.1002/joc.1971.

Yu, H.-L., G. Christakos, and P. Bogaert, 2010: Dealing with spatiotemporal heterogeneity: The generalized BME model. Progress in Spatial Analysis: Methods and Applications, A. Páez et al., Eds., Springer, 75-91. 